\title{
Role of Primary Care Optometrists in the Assessment and Management of Patients with Traumatic Brain Injuries in Canada
}

Zoe Lacroix, O.D.

Clinical Resident, Paediatrics and

Vision Therapy

School of Optometry and Vision Science, University of Waterloo

Susan J. Leat, PhD, FCOptom, F.A.A.O.

Professor, School of Optometry and Vision Science, University of Waterloo

Lisa W. Christian, O.D., F.C.O.V.D., F.A.A.O. Clinical Associate Professor School of Optometry and Vision Science, University of Waterloo Waterloo

\begin{abstract}
Introduction
Traumatic brain injury (TBI) results from a strong blow or jolt to the head that disrupts the normal function of the brain. ${ }^{1}$ The severity of a TBI can range from mild to severe, depending on the patient's mental status, consciousness level and amnesia following the injury. The annual incidence of TBI in North America and Europe is conservatively estimated to be approximately $600 / 100,000 .^{2,3}$ This translates to at least 200,000 TBI cases in Canada every year. According to the Centers for Disease Control and Prevention, and the Canadian Institute for Health Information, the leading cause of TBIs that result in hospital admission is falls (35\%-45\%), followed by motor vehicle accidents (17\%-36\%), collision-related events (struck by or against) (10-17\%) and assaults (9-10\%)., Head injuries are more common in the 0 - to 19-year age group, followed by those who are aged $60+$. Males are more highly represented in every age group than females. However, it should be noted that the demographics of patients who present in an optometrist's office may differ from those based on hospital admissions.
\end{abstract}

TBIs are classified by the duration of loss of consciousness and post-traumatic amnesia, along with the results of brain imaging (Table 1) ${ }^{6,7}$ Not all of these signs need to be present. Menon et al. stated that TBI can be diagnosed when there is alteration in brain function defined by any one of the following signs: a period of loss or decreased consciousness, any loss of memory for events immediately before or after the injury, neurologic deficits (weakness, loss of balance, change in vision, dyspraxia paresis/paralysis, sensory loss, aphasia, etc.), and any alteration in mental state at the time of the injury (confusion, disorientation, slowed thinking, etc.). ${ }^{8}$

Common phenomena following a TBI include decreased attention, concentration and processing speed, memory problems, confusion, irritability, depression and anxiety. Physical consequences can include headaches, fatigue, dizziness and nausea, balance difficulties, visual disturbance and sleep disruption. ${ }^{1}$ In cases of moderate to severe TBI, patients may also experience decreased executive function, increased confusion, depression, anxiety, lack of impulse control, chronic pain and severe physical consequences. ${ }^{6}$ Visual symptoms are observed in $75 \%$ of TBI cases. ${ }^{9}$ These symptoms can be caused by decreased visual function, disorders of the binocular vision system, changes in ocular health and higher-order processing disorders, which are discussed further below. Due to the broad spectrum of visual symptoms that may occur following TBIs, it is important for the primary care optometrist to be familiar with the testing and management of patients with a history of traumatic brain injury. 
Table 1: TBI Classification System ${ }^{6,7}$

\begin{tabular}{|l|l|l|l|}
\hline Characteristic & Mild TBI & Moderate TBI & Severe TBI \\
\hline Loss of consciousness (LOC) & 0 to 30 mins & 0.5 to $24 \mathrm{~h}$ & $>24 \mathrm{~h}$ \\
\hline Post-traumatic amnesia (PTA) & $0-1$ days & $1-7$ days & $>7$ days \\
\hline Brain-imaging results & Normal & Abnormal & Abnormal \\
\hline
\end{tabular}

\section{EVALUATION}

A thorough ocular-visual assessment (OVA) should be performed for all patients following a TBI. Testing includes a detailed case history, refraction, routine binocular vision and accommodation assessment, automated visual fields and ocular health assessment with dilated fundus evaluation. Additional in-depth testing of some systems should be included in the OVA for patients with a suspected or diagnosed TBI. This includes complementary assessments of the accommodative, vergence and oculomotor systems, and may include visual information-processing testing and visual-midline shift assessment.

The TBI population is also susceptible to cognitive and/or memory impairments, and special consideration should be given when considering the speed and duration of testing. Patients with TBI frequently require more time to process questions and commands. Therefore, objective measurements are preferred, as they will often provide more reliable results. ${ }^{10}$ The results of clinical testing should include any reported dizziness, headaches, nausea, or photophobia. If necessary, it may be beneficial to separate the vision examination into two or more appointments.

\section{Case history}

A thorough review of visual symptoms should be conducted, which can be facilitated by a symptom checklist completed by the patient prior to the appointment. ${ }^{11}$ Case history should include details of the TBI incident and associated injuries. It is useful to review the patient's current and previous rehabilitation services and the progress of their therapy (occupational therapy, physiotherapy, etc.). Patient goals and needs should also be assessed, including their occupational and vocational visual demands, computer use, driving, mobility and reading. Optometrists should remember to record previous ocular conditions and general health (pre- and post-TBI), to differentiate between new and pre-existing conditions.

\section{Visual acuity and refraction}

Visual acuity itself is less often affected by TBI, and therefore traditional methods (i.e. Snellen) can be used to assess visual acuity in TBI patients. If a patient has cognitive or communication impairments, modified charts such as a Tumbling E or Broken Wheel test may provide more valid results. ${ }^{11}$

When the optometrist performs a refraction, objective measurements such as retinoscopy should be considered for all patients since it may be difficult to elicit reliable subjective responses. Automated refractors can also be considered for photophobic patients. Although TBIs may not directly change a patient's refractive error, this population may become more sensitive to small prescription changes or uncorrected refractive errors. Special consideration should be given to latent or uncorrected hyperopic patients, who may become symptomatic following a TBI. ${ }^{12}$ Progressive addition lenses are not recommended due to peripheral distortions.

\section{Ocular health}

A thorough slit lamp examination is performed to assess the ocular health of TBI patients, including a dilated fundus evaluation. Ocular health disorders following TBI can affect the anterior or posterior segments and may include angle recession, dry eye, intraocular hemorrhage or embolisms and papilledema. ${ }^{13-17}$ An in-depth assessment of the cranial nerves, pupils and optic nerves should also be performed. Appropriate treatment should be made for the management of these conditions or referral when indicated, such as to the family physician, ophthalmologist, neuro-ophthalmologist, neurologist, etc.

\section{Visual field}

Visual field defects may occur through trauma to the optic nerve, chiasm, optic radiations, or occipital cortex..$^{15-17} \mathrm{Subtle}$ visual field defects may not be detected by confrontational visual field. ${ }^{18}$ Automated perimetry is better suited for de- 
tecting mild neurological defects and for monitoring changes over time. ${ }^{18}$ Screening for visual neglect should also be considered for TBI patients. ${ }^{19-21}$ Tests for spatial inattention (neglect) include linebisection and the clock drawing test. ${ }^{22}$

Once appropriate investigation into the visual defect has been completed, treatment therapies are typically aimed at increasing the awareness of the affected field and the development of compensatory techniques. This can be achieved via field-enhancing prisms such as sector prisms, or Peli prisms. ${ }^{23,24}$ These prisms are aimed at bringing the image of the affected field into view to provide the patient with information about their periphery. Compensatory functional and rehabilitative techniques can also be taught to patients, such as field scanning, and visuomotor, behavioural and reading techniques. ${ }^{24,25}$

\section{Visual midline shift}

Visual midline shift syndrome (VMSS) has been defined as a sense of shifted egocenter and has been reported after brain injuries. ${ }^{26}$ It is often associated with, and indeed, may result from, neglect and/or hemianopia, although the exact association has not been documented. These alterations in the perceived midline can create changes in balance and posture. Healthcare professionals who typically address gait and balance include physiotherapists and occupational therapists. ${ }^{26}$

Standardized assessment procedures have not been developed for visual midline shift testing. Current techniques include the subjective alignment of a wand at the midline, eye-hand coordination tests, observation of gait, as well as emerging devices to more accurately quantify the deviation and egocenter. ${ }^{26}$ Padula and Argyris stated that a horizontal shift in midline may result in a lateral lean away from the affected visual space, and a possible drift left or right when walking. A vertical shift may result in tilting the body forward or backward (posterior/anterior). ${ }^{27}$

Although further research in this field is needed, practitioners have reported success with the use of compensatory yoked prisms.$^{26}$ For assessment, prism lenses are initially placed with the base in the direction opposite the perceived shift in midline, aiming to realign the patient's egocenter. Testing is then repeated with different lenses in place. These trials are usually completed with yoked prisms under 10-12 prism diopters. Spatial localization therapies have also been used to enhance eye-hand coordination. A second approach is prism adaptation, in which localization training is undertaken with prisms in place, with the base contralateral to the direction of the shift. Typically, a higher power of prism is used (17 prism diopters). When the prisms are removed, pointing becomes more central, which can last up to 3.5 years. ${ }^{28}$

\section{Accommodation}

Accommodative dysfunctions are present in approximately $40 \%$ of TBI patients, ${ }^{29,30}$ and include accommodative insufficiency, accommodative infacility, or accommodative spasms (which may induce pseudo-myopia). ${ }^{31}$ Accommodative testing should include the assessment of accommodative amplitudes (push-up to blur, or pull away to clear), accommodative accuracy (Monocular Estimation Method, cross cylinder evaluation, or Nott's modified dynamic retinoscopy) and accommodative facility (monocular and binocular). ${ }^{10}$

Management of accommodative disorders may include reading glasses with increased plus at near, ${ }^{10}$ or vision rehabilitation exercises. ${ }^{10,32,33}$ In non-presbyopic patients, vision exercises are usually recommended as the initial treatment and may include accommodative rock using lenses or different distances, as well as accommodative push-up techniques. There is some evidence that $87-100 \%$ of patients with accommodative dysfunctions show improvements with vision therapy. ${ }^{33}$

\section{Binocular vision}

Vergence dysfunctions are one of the most common disorders following TBI, and are seen in approximately $50 \%$ of patients. ${ }^{9,29,34}$ Common disorders include convergence insufficiency (36\%), binocular instability (restricted vergence ranges) (10\%), basic esophoria (18\% of patients with cerebrovascular accidents) and strabismus (e.g., intermittent exotropia, cranial nerve palsy) (7-25\%)., 29,34

Binocular vision testing should include routine and additional testing, including ocular alignment at distance and near (cover test, Maddox rod, phoria, associated phoria), motor fusion (vergence ranges, near point of convergence, vergence facility with 3BI/12BO prism jumps), sensory fusion (stereoscopy and fusion) and ocular motilities. ${ }^{12}$ 
Management of vergence disorders may include lenses, correcting prism, or vision therapy exercises..$^{12,33,35}$ Vision therapy is usually recommended as the initial treatment for convergence insufficiency, while plus lenses should initially be considered for convergence excess. In-office binocular vision training has been used to successfully treat $>75 \%$ of TBI patients with convergence insufficiency. ${ }^{33,35}$ These therapies include Brock string, pencil pushups, prism jumps, or instruments such as the Aperture Rule, cheiroscopes, vectograms and tranaglyphs, often in combination.

\section{Oculomotor}

Fixation, pursuits and saccades are affected in approximately $20 \%$ of TBI patients. ${ }^{36}$ Test procedures that involve oculomotor function include the Developmental Eye Movement Test, King Devick Test, Visagraph/ReadAlyzer goggles with infra-red sensors and the NSUCO (Northeastern State University College of Optometry) and SCCO (Southern California College of Optometry) oculomotor tests. ${ }^{12}$

Treatment is aimed at training each of these individual skills. There is some evidence that oculomotor therapies are successful in improving these skills, especially with reading. ${ }^{12,33,37}$ Although training techniques for oculomotor skills have not been extensively researched, therapies can include letter-tracking workbooks, oculomotor pursuit exercises, Brock string fixations, flashlight tag and computerized programs (i.e., Home Therapy System [HTS]) or other computer-aided vision therapy software).

\section{Photophobia}

Following a TBI, patients commonly report photophobia and increased sensitivity to glare. ${ }^{38}$ Despite its prevalence, photophobia remains poorly understood and is difficult to assess and treat. Ongoing research in this field is performed to better understand the underlying mechanisms. Various theories have attributed photophobia to migraines following TBI, damage to the pain-sensitive intracranial structure and deficits in dark adaptation..$^{38-40}$

For TBI patients, the case history should include questions about increased sensitivity to glare, sunlight, computers and screens. ${ }^{41}$ Careful pupil testing should be performed, although this will often yield normal results. Dry eye or headaches should also be investigated, as these conditions may exacerbate photophobia symptoms. ${ }^{38}$ All underlying disease should be appropriately treated.

Although no major studies have been conducted on the management of photophobia symptoms, current treatment options include tinted lenses, overlays, and polarized, photochromic or fit-over sunglasses. ${ }^{41}$ These options are mostly selected subjectively, but often provide relief to patients and improve their visual comfort. However, there is some evidence that no tint, lighter tints or decreasing the tint over time encourages a decrease in photosensitivity with time. ${ }^{42}$

\section{MULTIDISCIPLINARY APPROACH}

It is not uncommon for TBI patients to have comorbid health conditions. A multidisciplinary approach is always recommended when managing these patients. Interprofessional collaboration with other health care providers allows improved patient care through regular progress reports and communications. In addition to optometrists and ophthalmologists, other specialists who are often involved in the care of TBI patients include medical doctors, neurologists, physiotherapists, occupational therapists, audiologists, vestibular therapists, physical therapists and chiropractors. It is recommended that optometrists develop a good relationship with other providers to ensure optimal patient care. Allied healthcare providers should be provided with a report detailing the oculo-visual findings and recommendations for mutual patients.

\section{CONCLUSION}

As discussed, visual symptoms are very common following TBI. These patients benefit from a thorough optometric evaluation to identify and manage any underlying vision condition. Treatments may include tinted lenses and overlays, corrective and prismatic lenses, and vision therapy and rehabilitation. Addressing the visual needs of patients with TBI can reduce their symptoms, improve their quality of life and help them return to work and daily living. $\bullet$ 


\section{REFERENCES}

1. Centers for Disease Control and Prevention (CDC), National Center for Injury Prevention and Control. Report to Congress on mild traumatic brain injury in the United States: steps to prevent a serious public health problem. Centers for Disease Control and Prevention. 2003.

2. Cassidy JD, Carroll LJ, Peloso PM, et al. Incidence, risk factors and prevention of mild traumatic brain injury: results of the WHO Collaborating Centre Task Force on Mild Traumatic Brain Injury. J Rehabil Med. 2004(43 Suppl):28-60.

3. Canadian Institutes of Health Research. Research in Traumatic Brain Injuries 2012 [Available from: http://www.cihr-irsc. gc.ca/e/45665.html]. Accessed 05/01/2017.

4. Centers for Disease Control and Prevention. Traumatic brain injury in the United States: emergency department visits, hospitalizations, and deaths, 2002-2006. Atlanta (GA); 2010.

5. Canadian Institute for Health Information. Head Injuries in Canada: A Decade of Change (1994-1995 to 2003-2004). 2006. [Available from: https://secure.cihi.ca/free_products/ntr_head_ injuries_2006_e.pdf]. Accessed 05/01/2017.

6. Centers for Disease Control and Prevention. Injury Prevention \& Control: Traumatic Brain Injury \& Concussion.

7. Blyth BJ, Bazarian JJ. Traumatic alterations in consciousness: traumatic brain injury. Emerg Med Clin North Am. 2010;28(3):571-94.

8. Menon DK, Schwab K, Wright DW, Maas AI. Position Statement: Definition of traumatic brain injury. Arch Phys Med Rehab 2010;91(11):1637-40.

9. Brahm KD, Wilgenburg HM, Kirby J, Ingalla S, Chang CY, Goodrich GL. Visual impairment and dysfunction in combat-injured servicemembers with traumatic brain injury. Optom Vis Sci. 2009;86(7):817-25.

10. Cohen A. Acquired visual information-processing disorders: closed head trauma. Applied Concepts in Vision Therapy, Press L, ed. St Louis: Mosby; 1997. p. 154-66.

11. Kara G, Chrystyna R. Examination Approach. 2013. In: Brain Injury Electronic Resource Manual [Available from: http://aoa.uberflip. com/i/225867-brain-injury-electronic-resource-manual]. American Optometric Association Vision Rehabilitation Section. Accessed 05/01/2017.

12. Scheiman M, Wick B. Binocular and Accommodative Problems Associated with Acquired Brain Injury. In: Clinical Management of Binocular Vision: Heterophoric, Accommodative and Eye Movement Disorders, 2nd ed. Philadelphia, PA: Lippincott, Williams and Wilkins; 2002, p. 571-592.

13. Cockerham GC, Lemke S, Glynn-Milley C, Zumhagen L, Cockerham $\mathrm{KP}$. Visual performance and the ocular surface in traumatic brain injury. Ocul Surf 2013;11 (1):25-34.

14. Candice E. Ocular Health Examination. 2013. In: Brain Injury Electronic Resource Manual [Available from: http://aoa.uberflip. com/i/225867-brain-injury-electronic-resource-manual]. American Optometric Association Vision Rehabilitation Section. Accessed 05/01/2017.

15. Atkins EJ, Newman NJ, Biousse V. Post-traumatic visual loss. Rev Neurol Dis 2008;5(2):73-81.

16. Jacobs SM, Van Stavern GP. Neuro-ophthalmic deficits after head trauma. Curr Neurol Neurosci Rep 2013;13(11):389.

17. Cockerham GC, Goodrich GL, Weichel ED, et al. Eye and visual function in traumatic brain injury. J Rehabil Res Dev 2009;46(6):811-8.

18. Kerr NM, Chew SS, Eady EK, Gamble GD, Danesh-Meyer HV. Diagnostic accuracy of confrontation visual field tests. Neurology 2010;74(15):1184-90.

19. Goodrich GL, Kirby J, Cockerham G, Ingalla SP, Lew HL. Visual function in patients of a polytrauma rehabilitation center: A descriptive study. J Rehabil Res Dev 2007;44(7):929-36.
20. Halterman CI, Langan J, Drew A, et al. Tracking the recovery of visuospatial attention deficits in mild traumatic brain injury. Brain 2006;129(3):747-53.

21. Suchoff IB, Ciuffreda KJ. A primer for the optometric management of unilateral spatial inattention. Optometry 2004;75(5):305-18.

22. Kara G, Chrystyna R, Brenda M, Michael P. Examination of Cortical Visual Function. 2013. In: Brain Injury Electronic Resource Manual [Available from: http://aoa.uberflip.com/i/ 225867- brain-injuryelectronic-resource-manual]. American Optometric Association Vision Rehabilitation Section. Accessed 05/01/2017.

23. Ross NC, Bowers AR, Peli E. Peripheral prism glasses: effects of dominance, suppression, and background. Optom Vis Sci 2012;89(9):1343-52.

24. Kapoor N, Ciuffreda KJ. Vision disturbances following traumatic brain injury. Curr Treat Options Neurol 2002;4(4):271-80.

25. Goodwin D. Homonymous hemianopia: challenges and solutions. Clin Ophthalmol (Auckland, NZ). 2014;8:1919-27.

26. Padula WV, Nelson CA, Benabib R, Yilmaz T, Krevisky S. Modifying postural adaptation following a CVA through prismatic shift of visuo-spatial egocenter. Brain Inj 2009;23(6):566-76.

27. Padula WV, Argyris S. Post trauma vision syndrome and visual midline shift syndrome. NeuroRehabilitation 1996;6(3):165-71.

28. Keane S, Turner C, Sherrington C, Beard JR. Use of fresnel prism glasses to treat stroke patients with hemispatial neglect. Arch Phys Med Rehabil 2006;87:1668-72.

29. Ciuffreda KJ, Kapoor N, Rutner D, Suchoff IB, Han ME, Craig S. Occurrence of oculomotor dysfunctions in acquired brain injury: a retrospective analysis. Optometry 2007;78(4):155-61.

30. Green W, Ciuffreda KJ, Thiagarajan P, Szymanowicz D, Ludlam DP, Kapoor N. Accommodation in mild traumatic brain injury. J Rehabil Res Dev 2010;47(3):183-99.

31. London R, Wick B, Kirschen D. Post-traumatic pseudomyopia. Optometry 2003;74(2):111-20.

32. Scheiman M, Cotter S, Kulp MT, et al. Treatment of accommodative dysfunction in children: results from a randomized clinical trial. Optom Vis Sci 2011;88(11):1343-52.

33. Ciuffreda KJ, Rutner D, Kapoor N, Suchoff IB, Craig S, Han ME. Vision therapy for oculomotor dysfunctions in acquired brain injury: a retrospective analysis. Optometry 2008;79(1):18-22.

34. Capo-Aponte JE, Urosevich TG, Temme LA, Tarbett AK, Sanghera NK. Visual dysfunctions and symptoms during the subacute stage of blast-induced mild traumatic brain injury. Mil Med 2012;177(7):80413.

35. Scheiman M, Mitchell GL, Cotter S, et al. A randomized clinical trial of treatments for convergence insufficiency in children. Arch Ophthalmo. 2005;123(1):14-24.

36. Hunt AW, Mah K, Reed N, Engel L, Keightley M. Oculomotor-based vision assessment in mild traumatic brain injury: a systematic review. J Head Trauma Rehabil 2016;31(4):252-61.

37. Ciuffreda KJ, Han Y, Kapoor N, Ficarra AP. Oculomotor rehabilitation for reading in acquired brain injury. NeuroRehabilitation 2006;21(1):9-21.

38. Digre KB, Brennan KC. Shedding light on photophobia. J Neuroophthalmol 2012;32(1):68-81.

39. Du T, Ciuffreda KJ, Kapoor N. Elevated dark adaptation thresholds in traumatic brain injury. Brain Inj 2005;19(13):1125-38.

40. Nampiaparampil DE. Prevalence of chronic pain after traumatic brain injury: a systematic review. JAMA 2008;300(6):711-9.

41. Barnett B, Singman E. Vision concerns after mild traumatic brain injury. Curr Treat Options Neurol 2015.17(2):329.

42. Truong JQ, Ciuffreda KJ, Han MH, Suchoff IB. Photosensitivity in mild traumatic brain injury (mTBI): A retrospective analysis. Brain Inj 2014;28(10):1283-7. 